\title{
Some integral inequalities for approximately $h$-convex functions and their applications
}

\author{
Artion Kashuri ${ }^{1} @$ orcid.org/0000-0003-0115-3079 \\ Muhammad Raees ${ }^{2}$ \\ Matloob Anwar ${ }^{3}$ [D orcid.org/0000-0001-5649-9769
}

${ }^{1}$ University of Vlorë "Ismail Qemali”, Dept. of Mathematics, Vlorë, Albania.

artionkashuri@gmail.com

National University of Sciences \& Technology, School of Natural Sciences, Islamabad, Pakistan.

2®muhammad.raees@sns.nust.edu.pk; ${ }^{3}{ }^{1}$ manwar@sns.nust.edu.pk

Received: May 2020 | Accepted: October 2020

\section{Abstract:}

In this paper, by applying the new and improved form of Hölder's integral inequality called Hölder-Iscan integral inequality three inequalities of Hermite-Hadamard and Hadamard integral type for $(h, d)$-convex functions have been established. Various special cases including classes for instance, $h$ convex, s-convex function of Breckner and Godunova-Levin-Dragomir and strong versions of the aforementioned types of convex functions have been identified. Some applications to error estimations of presented results have been analyzed. At the end, a briefly conclusion is given.

Keywords: Hermite-Hadamard inequality; Hölder-Íşcan inequality; Convexity, Numerical integration.

MSC (2020): 26A51, 26A33, 26D07, 26D10, 26D15

\section{Cite this article as (IEEE citation style):}

A. Kashuri, M. Raees, and M. Anwar, "Some integral inequalities for approximately $h$-convex functions and their applications", Proyecciones (Antofagasta, On line), vol. 40, no. 2, pp. 481-504, 2021, doi: 10.22199/issn.0717-6279-2021-020028

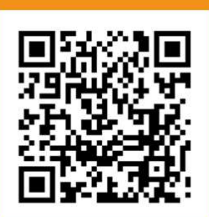

Article copyright: (C) 2021 Artion Kashuri, Muhammad Raees and Matloob Anwar. This is an open access article distributed under the terms of the Creative Commons License, which permits unrestricted use and distribution provided the original author and source are credited. 


\section{Introduction}

Convex functions and its generalizations have various applications in the fields of pure and applied sciences. Due to these applications, it is the most attractive area for researchers now a days. The class of convex functions is well known in the literature and is usually defined in the following way:

Definition 1.1. Let $I$ be an interval in $\mathbf{R}$. A function $\varrho: I \rightarrow \mathbf{R}$, is said to be convex on $I$ if the inequality

$$
\varrho\left(u a_{1}+(1-u) a_{2}\right) \leq u \varrho\left(a_{1}\right)+(1-u) \varrho\left(a_{2}\right)
$$

holds for all $a_{1}, a_{2} \in I$ and $u \in[0,1]$. Also, we say that $\varrho$ is concave, if the inequality in (1.1) holds in the reverse direction.

Theorem 1.2. Let $\varrho: I \subset \mathbf{R} \longrightarrow \mathbf{R}$ be a convex function, $a_{1}, a_{2} \in I$ and $a_{1}<a_{2}$. Then

$$
\varrho\left(\frac{a_{1}+a_{2}}{2}\right) \leq \frac{1}{a_{2}-a_{1}} \int_{a_{1}}^{a_{2}} \varrho(u) d u \leq \frac{\varrho\left(a_{1}\right)+\varrho\left(a_{2}\right)}{2} .
$$

Authors of recent decades have studied the inequality (1.2), known as Hermite-Hadamard inequality (or trapezium inequality) in the premises of newly invented definitions due to motivation of convex function. Interested readers can see the references $[2,3,4,5,6,7,8,9,10,11$, $12,13,14,15,16,17,18,19,20,21,22,23,24,25,26,27,28,29,29,30,31]$.

The stability theory for functional inequalities has its origin in the paper of Hyers and Ulam [13], in which the notion of $\delta$-convex functions is introduced. A function $\varrho: D \rightarrow \mathbf{R}$ is known as $\delta$-convex function, if

$$
\varrho\left(u a_{1}+(1-u) a_{2}\right) \leq u \varrho\left(a_{1}\right)+(1-u) \varrho\left(a_{2}\right)+\delta
$$

for all $a_{1}, a_{2} \in D, u \in[0,1]$, where $D$ is a convex subset of a real linear space $X$ and $\delta$ is a non-negative real number. Roughly speaking, the HyersUlam theorem says that a function $\varrho$ is indeed a perturbation of a convex function by a bounded function if and only if there exists a non negative real number $\delta$ which satisfies (1.3). Now the error term is now refer to a bounded perturbation. Páles in [24], extended the notion to $(\epsilon, \delta)$-convex functions while investigating the stability constants as follows:

A real valued function $\varrho$ defined on a real interval $I$ is called $(\epsilon, \delta)$-convex function if it satisfies the following inequality: 


$$
\varrho\left(u a_{1}+(1-u) a_{2}\right) \leq u \varrho\left(a_{1}\right)+(1-u) \varrho\left(a_{2}\right)+\epsilon u(1-u)\left\|a_{1}-a_{2}\right\|+\delta,
$$

$\forall a_{1}, a_{2} \in I$ and $u \in[0,1]$. In (1.4), there are two error terms. Páles in [24], shown that the error terms $\epsilon u(1-u)\left\|a_{1}-a_{2}\right\|$ and $\delta$, respectively, corresponds to perturbation of a convex function by a Lipschitz function and by a bounded function.

Burai and Hazy [5] considered a function $d: X \times X \rightarrow \mathbf{R}$ a function on normed space $X$ and $D$ a non-empty open, convex subset of $X$. They generalized the notion of convex function by introducing $(h, d)$-convex function as follows:

Definition 1.3. ( $(h, d)$-convex function) A function $\varrho: D \rightarrow \mathbf{R}$, is called $(h, d)$-convex function, if

$\varrho\left(u a_{1}+(1-u) a_{2}\right) \leq h(u) \varrho\left(a_{1}\right)+h(1-u) \varrho\left(a_{2}\right)+d\left(a_{1}, a_{2}\right), \quad \forall a_{1}, a_{2} \in D, u \in(0,1)$.

the class of $(h, d)$-convex functions includes the class of classical convex functions, $\delta$-convex functions, $(\epsilon, \delta)$-convex functions, $h$-convex functions, $s$-convex functions of Breckner type, $s$-convex functions of GodunovaLevin-Dragomir type etc., and classes of strongly convex versions of aforementioned convex functions.

It is worth mentioning that the convexity of the function $\varrho$ depends on the value of the function $d(\cdot, \cdot)$ and the function $d(\cdot, \cdot)$ entirely depends on the convex combination. Therefore it will be convenient to modify this definition. We know propose a modification to $(h, d)$-convex function.

Definition 1.4. ( Modified $(h, d)$-convex function) A function $\varrho$ : $D \rightarrow \mathbf{R}$, is called modified $(h, d)$-convex function, if

$$
\varrho\left(u a_{1}+(1-u) a_{2}\right) \leq h(u) \varrho\left(a_{1}\right)+h(1-u) \varrho\left(a_{2}\right)+u(1-u) d\left(a_{1}, a_{2}\right)
$$

$$
\forall a_{1}, a_{2} \in D, u \in(0,1) .
$$

Now we discuss some special cases, by restricting ourselves to the only well studied notions of convex functions by assigning different values to the function $d$.

I. If $d\left(a_{1}, a_{2}\right)=\frac{\delta}{u(1-u)}$, then we have following extension of well known $\delta$-convex functions given by inequality (1.3). 
Definition 1.5. ( $\delta-h-$ convex function) $A$ function $\varrho: D \rightarrow \mathbf{R}$ is called $\delta$ - $h$-convex function, if $\varrho$ satisfies

$$
\varrho\left(u a_{1}+(1-u) a_{2}\right) \leq h(u) \varrho\left(a_{1}\right)+h(1-u) \varrho\left(a_{2}\right)+\delta, \quad \forall a_{1}, a_{2} \in D, u \in(0,1) .
$$

Moreover we have class of $\delta$-convex functions, $\delta$-Godunova-Levin functions, $\delta$ - $P$-functions, $\delta$-tgs-functions, $\delta$-MT-convex functions, Breckner $\delta$ $s$-convex functions and $\delta$-s-Godunova-Levin functions by using $h(u)=$ $u, u^{-1}, 1, u(1-u), \frac{\sqrt{u}}{2 \sqrt{1-u}}, u^{s}$ and $h(u)=u^{-s}$, respectively.

II. If $d\left(a_{1}, a_{2}\right)=\epsilon\left\|a_{1}-a_{2}\right\|+\frac{\delta}{u(1-u)}$, then we have following new type of convex functions.

Definition 1.6. ( $(\epsilon, \delta)$ - $h$-convex function) A function $\varrho: D \rightarrow \mathbf{R}$ is called $(\epsilon, \delta)$-h-convex function if

$$
\begin{aligned}
& \text { 8) } \varrho\left(u a_{1}+(1-u) a_{2}\right) \leq h(u) \varrho\left(a_{1}\right)+h(1-u) \varrho\left(a_{2}\right)+\epsilon u(1-u)\left\|a_{1}-a_{2}\right\|+\delta, \\
& \forall a_{1}, a_{2} \in D \text { and } u \in(0,1) .
\end{aligned}
$$

Moreover we havee class of $(\epsilon, \delta)$-convex functions, $(\epsilon, \delta)$-Godunova-Levin functions, $(\epsilon, \delta)$-P-functions, $(\epsilon, \delta)$-tgs-functions, $(\epsilon, \delta)$-MT-convex functions, Breckner $(\epsilon, \delta)$-s-convex functions and $(\epsilon, \delta)$-s-Godunova-Levin functions by using $h(u)=u, u^{-1}, 1, u(1-u), \frac{\sqrt{u}}{2 \sqrt{1-u}}, u^{s}$ and $h(u)=u^{-s}$ respectively.

III. If $d\left(a_{1}, a_{2}\right)=-\epsilon\left\|a_{1}-a_{2}\right\|^{2}$, then we have following new type of convex functions.

Definition 1.7. (Strongly- $h$-convex function) A function $\varrho: D \rightarrow \mathbf{R}$ is called strongly $h$-convex function, if

$$
\varrho\left(u a_{1}+(1-u) a_{2}\right) \leq h(u) \varrho\left(a_{1}\right)+h(1-u) \varrho\left(a_{2}\right)-\epsilon u(1-u)\left\|a_{1}-a_{2}\right\|^{2},
$$

$$
\forall a_{1}, a_{2} \in D \text { and } u \in(0,1) .
$$


Moreover, classes of strongly convex functions, strongly Godunova-Levin functions, strongly $P$-functions, strongly $t g s$-functions, strongly $M T$-convex functions, Breckner strongly $s$-convex functions and strongly $s$-GodunovaLevin functions are obtained by using $h(u)=u, u^{-1}, 1, u(1-u), \frac{\sqrt{u}}{2 \sqrt{1-u}}, u^{s}$ and $h(u)=u^{-s}$, respectively.

Now we give some preliminaries which will be helpful for further study.

Sarikaya et al. [25] prove the following Hadamard inequality for $h$-convex function.

Theorem 1.8. Let $\varrho: I \rightarrow \mathbf{R}$ be an $h$-convex function, $a_{1}, a_{2} \in I$ with $a_{1}<a_{2}$ and $\varrho \in L_{1}\left[a_{1}, a_{2}\right]$. Then

$$
\frac{1}{2 h\left(\frac{1}{2}\right)} \varphi\left(\frac{a_{1}+a_{2}}{2}\right) \leq \frac{1}{a_{2}-a_{1}} \int_{a_{1}}^{a_{2}} \varrho(x) d x \leq\left[\varrho\left(a_{1}\right)+\varrho\left(a_{2}\right)\right] \int_{0}^{1} h(r) d r,
$$

which was proved in [1].

In [10], following identity for differentiable mappings is proved.

Lemma 1.9. Let $\varrho: I \subset \mathbf{R} \rightarrow \mathbf{R}$ be a differentiable function on $I^{0}$ where $a_{1}, a_{2} \in I$ with $a_{1}<a_{2}$. If $\varrho^{\prime} \in L\left[a_{1}, a_{2}\right]$, then the following equality holds:

$$
\frac{\varrho\left(a_{1}\right)+\varrho\left(a_{2}\right)}{2}-\frac{1}{a_{2}-a_{1}} \int_{a_{1}}^{a_{2}} \varrho(x) d x=\left(\frac{a_{2}-a_{1}}{2}\right) \int_{0}^{1}(1-2 u) \varrho^{\prime}\left(u a_{1}+(1-u) a_{2}\right) d u .
$$

The well-known Hölder inequality and power-mean inequalities are given as follows:

Theorem 1.10. ( Hölder integral inequality) Let $p>1$ and $\frac{1}{p}+\frac{1}{q}=1$. If $\phi$ and $\psi$ are real valued functions on $\left[a_{1}, a_{2}\right]$ and if $|\phi|^{p}$ and $|\psi|^{q}$ are integrable on $\left[a_{1}, a_{2}\right]$, then 


$$
\int_{a_{1}}^{a_{2}}|\phi(x) \psi(x)| d x \leq\left(\int_{a_{1}}^{a_{2}}|\phi(x)|^{p} d x\right)^{\frac{1}{p}}\left(\int_{a_{1}}^{a_{2}}|\psi(x)|^{q} d x\right)^{\frac{1}{q}}
$$

where equality holds if and only if $A|\phi(x)|^{p}=B|\psi(x)|^{q}$ almost everywhere, where $A$ and $B$ are constants.

Theorem 1.11. ( Power-mean integral inequality) Let $q \geq 1$. If $\phi$ and $\psi$ are real valued functions on $\left[a_{1}, a_{2}\right]$ and if $|\phi|$ and $|\phi||\psi|^{q}$ are integrable on $\left[a_{1}, a_{2}\right]$, then

$$
\int_{a_{1}}^{a_{2}}|\phi(x) \psi(x)| d x \leq\left(\int_{a_{1}}^{a_{2}}|\phi(x)| d x\right)^{1-\frac{1}{q}}\left(\int_{a_{1}}^{a_{2}}|\phi(x)||\psi(x)|^{q} d x\right)^{\frac{1}{q}} .
$$

Recently, in [14], Íşcan gave the following inequality which gives the refinement form of Hölder's integral inequality as follows:

Theorem 1.12. (Hölder-Íşcan integral inequality) Let $p>1$ and $\frac{1}{p}+\frac{1}{q}=1$. If $\phi$ and $\psi$ are real valued functions on $\left[a_{1}, a_{2}\right]$ and if $|\phi|^{p}$ and $|\psi|^{q}$ are integrable on $\left[a_{1}, a_{2}\right]$, then

$$
\begin{aligned}
& \int_{a_{1}}^{a_{2}}|\phi(x) \psi(x)| d x \\
& \leq \frac{1}{a_{2}-a_{1}}\left\{\left(\int_{a_{1}}^{a_{2}}\left(a_{2}-x\right)|\phi(x)|^{p} d x\right)^{\frac{1}{p}}\left(\int_{a_{1}}^{a_{2}}\left(a_{2}-x\right)|\psi(x)|^{q} d x\right)^{\frac{1}{q}}\right. \\
& \left.+\left(\int_{a_{1}}^{a_{2}}\left(x-a_{1}\right)|\phi(x)|^{p} d x\right)^{\frac{1}{p}}\left(\int_{a_{1}}^{a_{2}}\left(x-a_{1}\right)|\psi(x)|^{q} d x\right)^{\frac{1}{q}}\right\}
\end{aligned}
$$

$$
\leq\left(\int_{a_{1}}^{a_{2}}|\phi(x)|^{p} d x\right)^{\frac{1}{p}}\left(\int_{a_{1}}^{a_{2}}|\psi(x)|^{q} d x\right)^{\frac{1}{q}},
$$

where equality holds if and only if $A|\phi(x)|^{p}=B|\psi(x)|^{q}$ almost everywhere, where $A$ and $B$ are constants. 
A different version named as improved power-mean integral inequality can be given by:

Theorem 1.13. (Improved Power-mean integral inequality) Let $q \geq$ 1. If $\phi$ and $\psi$ are real valued functions on $\left[a_{1}, a_{2}\right]$ and if $|\phi|$ and $|\phi||\psi|^{q}$ are integrable on $\left[a_{1}, a_{2}\right]$, then

$$
\begin{gathered}
\int_{a_{1}}^{a_{2}}|\phi(x) \psi(x)| d x \\
\leq \frac{1}{a_{2}-a_{1}}\left\{\left(\int_{a_{1}}^{a_{2}}\left(a_{2}-x\right)|\phi(x)| d x\right)^{1-\frac{1}{q}}\left(\int_{a_{1}}^{a_{2}}\left(a_{2}-x\right)|\phi(x)||\psi(x)|^{q} d x\right)^{\frac{1}{q}}\right. \\
\left.+\left(\int_{a_{1}}^{a_{2}}\left(x-a_{1}\right)|\phi(x)| d x\right)^{1-\frac{1}{q}}\left(\int_{a_{1}}^{a_{2}}\left(x-a_{1}\right)|\phi(x)||\psi(x)|^{q} d x\right)^{\frac{1}{q}}\right\} \\
\leq\left(\int_{a_{1}}^{a_{2}}|\phi(x)| d x\right)^{1-\frac{1}{q}}\left(\int_{a_{1}}^{a_{2}}|\phi(x)||\psi(x)|^{q} d x\right)^{\frac{1}{q}} \cdot
\end{gathered}
$$

The main objective of this paper is to discover in Section 2, three inequalities of Hermite-Hadamard and Hadamard integral type for $(h, d)$-convex functions by applying Hölder-Íşcan integral inequalities. Various special cases including classes for instance, $h$-convex, $s$-convex function of Breckner and Godunova-Levin-Dragomir and strong versions of the aforementioned types of convex functions will be identified. In Section 3, some applications to special means and error estimations will be provided. In Section 4, a briefly conclusion is given as well.

\section{Main Results}

We have the following results:

Theorem 2.1. Let $\varrho$ be an integrable function defined on $\left[a_{1}, a_{2}\right]$. If $\varrho$ is a modified $(h, d)$-convex function on $\left[a_{1}, a_{2}\right]$, then the following inequality holds: 


$$
\begin{aligned}
& \frac{1}{2 h\left(\frac{1}{2}\right)}\left[\varrho\left(\frac{a_{1}+a_{2}}{2}\right)-\frac{1}{4} \int_{0}^{1} d\left(s a_{1}+(1-s) a_{2},(1-s) a_{1}+s a_{2}\right) d s\right] \\
& \leq \int_{0}^{1} \varrho\left(s a_{1}+(1-s) a_{2}\right) d s \\
(2.1) \leq & {\left[\varrho\left(a_{1}\right)+\varrho\left(a_{2}\right)\right] \int_{0}^{1} h(s) d s+\frac{1}{6} d\left(a_{1}, a_{2}\right) . }
\end{aligned}
$$

Proof. By the notion of modified $(h, d)$-convex functions, we have

$$
\varrho\left(\frac{x_{1}+x_{2}}{2}\right) \leq h\left(\frac{1}{2}\right)\left[\varrho\left(x_{1}\right)+\varrho\left(x_{2}\right)\right]+\frac{1}{4} d\left(x_{1}, x_{2}\right)
$$

for all $x_{1}, x_{2} \in\left[a_{1}, a_{2}\right]$. Now, by taking $x_{1}=s a_{1}+(1-s) a_{2}$ and $x_{2}=$ $s a_{2}+(1-s) a_{1}, s \in[0,1]$, from $(2.2)$, we get

$$
\begin{gathered}
\frac{1}{h\left(\frac{1}{2}\right)}\left[\varrho\left(\frac{a_{1}+a_{2}}{2}\right)-\frac{1}{4} d\left(s a_{1}+(1-s) a_{2},(1-s) a_{1}+s a_{2}\right)\right] \\
\leq \varrho\left(s a_{1}+(1-s) a_{2}\right)+\varrho\left((1-s) a_{1}+s a_{2}\right) .
\end{gathered}
$$

Now integrating $(2.3)$ over $(0,1)$ with respect to $s$, we obtain

$$
\begin{aligned}
& \frac{1}{2 h\left(\frac{1}{2}\right)}\left[\varrho\left(\frac{a_{1}+a_{2}}{2}\right)-\frac{1}{4} \int_{0}^{1} d\left(s a_{1}+(1-s) a_{2},(1-s) a_{1}+s a_{2}\right) d x\right] \\
& \leq \frac{1}{a_{2}-a_{1}} \int_{a_{1}}^{a_{2}} \varrho(x) d x .
\end{aligned}
$$

On the other hand, since $\varrho$ is an $(h, d)$-convex function, one has

$$
\begin{array}{r}
\frac{1}{a_{2}-a_{1}} \int_{a_{1}}^{a_{2}} \varrho(x) d x=\int_{0}^{1} \varrho\left(s a_{1}+(1-s) a_{2}\right) d s \leq\left[\varrho\left(a_{1}\right)+\varrho\left(a_{2}\right)\right] \\
\int_{0}^{1} h(s) d s+\frac{1}{6} d\left(a_{1}, a_{2}\right) .
\end{array}
$$


Required double inequality (2.1) is obtained by combining (2.4) and (2.5).

Remark 2.2. If $d(x, y)=0$, then we obtain [25, Theorem 6].

Corollary 2.3. If $\varrho$ is an $\delta$ - $h$-convex function on $\left[a_{1}, a_{2}\right]$, then

$\frac{1}{2 h\left(\frac{1}{2}\right)}\left[\varrho\left(\frac{a_{1}+a_{2}}{2}\right)-\delta\right] \leq \frac{1}{a_{2}-a_{1}} \int_{a_{1}}^{a_{2}} \varrho(x) d x \leq\left[\varrho\left(a_{1}\right)+\varrho\left(a_{2}\right)\right] \int_{0}^{1} h(s) d s+\delta$.

Corollary 2.4. If $\varrho$ is $(\epsilon, \delta)$ - $h$-convex function on $\left[a_{1}, a_{2}\right]$, then

$$
\begin{aligned}
& \frac{1}{2 h\left(\frac{1}{2}\right)}\left[\varrho\left(\frac{a_{1}+a_{2}}{2}\right)-\delta\right] \leq \frac{1}{a_{2}-a_{1}} \int_{a_{1}}^{a_{2}} \varrho(x) d x \\
& \leq\left[\varrho\left(a_{1}\right)+\varrho\left(a_{2}\right)\right] \int_{0}^{1} h(s) d s+\frac{\epsilon}{6}\left(a_{2}-a_{1}\right)+\delta .
\end{aligned}
$$

Corollary 2.5. If $\varrho$ is $(\epsilon, \delta)$-s-convex function of Breckner type on $\left[a_{1}, a_{2}\right]$, then

$$
\begin{aligned}
2^{s-1} & {\left[\varrho\left(\frac{a_{1}+a_{2}}{2}\right)-\delta\right] \leq \frac{1}{a_{2}-a_{1}} \int_{a_{1}}^{a_{1}} \varrho(x) d x } \\
& \leq \frac{\varrho\left(a_{1}\right)+\varrho\left(a_{2}\right)}{s+1}+\frac{\epsilon}{6}\left(a_{2}-a_{1}\right)+\delta .
\end{aligned}
$$

Corollary 2.6. If $\varrho$ is $(\epsilon, \delta)$-s-convex function of Godunova-Levin-Dragomir type on $\left[a_{1}, a_{2}\right]$, then

$$
\begin{aligned}
& 2^{-(s+1)}\left[\varrho\left(\frac{a_{1}+a_{2}}{2}\right)-\delta\right] \leq \frac{1}{a_{2}-a_{1}} \int_{a_{1}}^{a_{2}} \varrho(x) d x \\
& \leq \frac{\varrho\left(a_{1}\right)+\varrho\left(a_{2}\right)}{1-s}+\frac{\epsilon}{6}\left(a_{2}-a_{1}\right)+\delta .
\end{aligned}
$$

Corollary 2.7. If $\varrho$ is $(\epsilon, \delta)$-P-convex function on $\left[a_{1}, a_{2}\right]$, then

$$
\begin{gathered}
\frac{1}{2}\left[\varrho\left(\frac{a_{1}+a_{2}}{2}\right)-\delta\right] \leq \frac{1}{a_{2}-a_{1}} \int_{a_{1}}^{a_{2}} \varrho(x) d x \\
\quad \leq\left[\varrho\left(a_{1}\right)+\varrho\left(a_{2}\right)\right]+\frac{\epsilon}{6}\left(a_{2}-a_{1}\right)+\delta .
\end{gathered}
$$


Corollary 2.8. If $\varrho$ is strongly- $h$-convex function on $\left[a_{1}, a_{2}\right]$, then

$$
\begin{gathered}
\frac{1}{2 h\left(\frac{1}{2}\right)}\left[\varrho\left(\frac{a_{1}+a_{2}}{2}\right)+\frac{\epsilon}{12}\left(a_{2}-a_{1}\right)^{2}\right] \leq \frac{1}{a_{2}-a_{1}} \int_{a_{1}}^{a_{2}} \varrho(x) d x \\
\quad \leq\left[\varrho\left(a_{1}\right)+\varrho\left(a_{2}\right)\right] \int_{0}^{1} h(s) d s-\frac{\epsilon}{6}\left(a_{2}-a_{1}\right)^{2},
\end{gathered}
$$

which was proved in [1].

Corollary 2.9. If $\varrho$ is strongly $s$-convex function of Breckner type on $\left[a_{1}, a_{2}\right]$, then

$$
\begin{gathered}
2^{s-1}\left[\varrho\left(\frac{a_{1}+a_{2}}{2}\right)+\frac{\epsilon}{12}\left(a_{2}-a_{1}\right)^{2}\right] \leq \frac{1}{a_{2}-a_{1}} \int_{a_{1}}^{a_{2}} \varrho(x) d x \\
\leq \frac{\varrho\left(a_{1}\right)+\varrho\left(a_{2}\right)}{s+1}+\frac{\epsilon}{6}\left(a_{2}-a_{1}\right)^{2} .
\end{gathered}
$$

Corollary 2.10. If $\varrho$ is strongly $\delta$-s-convex function of Godunova-LevinDragomir type on $\left[a_{1}, a_{2}\right]$, then

$$
\begin{gathered}
2^{-(s+1)}\left[\varrho\left(\frac{a_{1}+a_{2}}{2}\right)+\frac{\epsilon}{12}\left(a_{2}-a_{1}\right)^{2}\right] \leq \frac{1}{a_{2}-a_{1}} \int_{a_{1}}^{a_{2}} \varrho(x) d x \\
\leq \frac{\varrho\left(a_{1}\right)+\varrho\left(a_{2}\right)}{1-s}+\frac{\epsilon}{6}\left(a_{2}-a_{1}\right)^{2} .
\end{gathered}
$$

Corollary 2.11. If $\varrho$ is strongly $P$-convex function on $\left[a_{1}, a_{2}\right]$, then

$$
\begin{gathered}
\frac{1}{2}\left[\varrho\left(\frac{a_{1}+a_{2}}{2}\right)+\frac{\epsilon}{12}\left(a_{2}-a_{1}\right)^{2}\right] \leq \frac{1}{a_{2}-a_{1}} \int_{a_{1}}^{a_{2}} \varrho(x) d x \leq\left[\varrho\left(a_{1}\right)+\varrho\left(a_{2}\right)\right] \\
+\frac{\epsilon}{6}\left(a_{2}-a_{1}\right)^{2} .
\end{gathered}
$$

Theorem 2.12. Let $\varrho$ be a differentiable function defined on $\left[a_{1}, a_{2}\right]$. If $\left|\varrho^{\prime}\right|^{q}$ is a modified $(h, d)$-convex function on $\left[a_{1}, a_{2}\right]$, where $p>1$ and $\frac{1}{p}+\frac{1}{q}=$ 1 , then the following inequality holds: 


$$
\begin{aligned}
& \left|\frac{\varrho\left(a_{1}\right)+\varrho\left(a_{2}\right)}{2}-\frac{1}{a_{2}-a_{1}} \int_{a_{1}}^{a_{2}} \varrho(x) d x\right| \leq \frac{\left(a_{2}-a_{1}\right)}{2 \sqrt[p]{2(p+1)}} \\
& \times\left\{\left[H_{1}\left|\varrho^{\prime}\left(a_{1}\right)\right|^{q}+H_{2}\left|\varrho^{\prime}\left(a_{2}\right)\right|^{q}+\frac{1}{12} d\left(a_{1}, a_{2}\right)\right]^{\frac{1}{q}}\right. \\
& \left.+\left[H_{1}\left|\varrho^{\prime}\left(a_{2}\right)\right|^{q}+H_{2}\left|\varrho^{\prime}\left(a_{1}\right)\right|^{q}+\frac{1}{12} d\left(a_{1}, a_{2}\right)\right]^{\frac{1}{q}}\right\},
\end{aligned}
$$

where

$$
\mathrm{H}_{1}=\int_{0}^{1} u h(u) d u, \quad H_{2}=\int_{0}^{1} u h(1-u) d u .
$$

Proof. Taking the modulus on both sides of identity (1.11) and using Hölder-Íşcan integral inequality, we have

$$
\begin{gathered}
\left|\frac{\varrho\left(a_{1}\right)+\varrho\left(a_{2}\right)}{2}-\frac{1}{a_{2}-a_{1}} \int_{a_{1}}^{a_{2}} \varrho(x) d x\right| \\
\leq\left(\frac{a_{2}-a_{1}}{2}\right) \int_{0}^{1}|1-2 u|\left|\varrho^{\prime}\left(u a_{1}+(1-u) a_{2}\right)\right| d u \\
\leq\left(\frac{a_{2}-a_{1}}{2}\right)\left\{\left(\int_{0}^{1}(1-u)|1-2 u|^{p} d u\right)^{\frac{1}{p}}\left(\int_{0}^{1}(1-u)\left|\varrho^{\prime}\left(u a_{1}+(1-u) a_{2}\right)\right|^{q} d u\right)^{\frac{1}{q}}\right. \\
\left.(2.7)+\left(\int_{0}^{1} u|1-2 u|^{p} d u\right)^{\frac{1}{p}}\left(\int_{0}^{1} u\left|\varrho^{\prime}\left(u a_{1}+(1-u) a_{2}\right)\right|^{q} d u\right)^{\frac{1}{q}}\right\} .
\end{gathered}
$$

Since $\left|\varrho^{\prime}\right|^{q}$ is modified $(h, d)$-convex function on $\left[a_{1}, a_{2}\right]$, we get

$$
\begin{array}{r}
\int_{0}^{1} u\left|\varrho^{\prime}\left(u a_{1}+(1-u) a_{2}\right)\right|^{q} d u \\
\leq \int_{0}^{1} u\left[h(u)\left|\varrho^{\prime}\left(a_{1}\right)\right|^{q}+h(1-u)\left|\varrho^{\prime}\left(a_{2}\right)\right|^{q}+u(1-u) d\left(a_{1}, a_{2}\right)\right] d u \\
=\int_{0}^{1} u h(u)\left|\varrho^{\prime}\left(a_{1}\right)\right|^{q} d u+\int_{0}^{1} u h(1-u)\left|\varrho^{\prime}\left(a_{2}\right)\right|^{q} d u+\frac{1}{12} d\left(a_{1}, a_{2}\right)
\end{array}
$$


and

$$
\begin{gathered}
\int_{0}^{1}(1-u)\left|\varrho^{\prime}\left(u a_{1}+(1-u) a_{2}\right)\right|^{q} d u \leq \int_{0}^{1} u h(1-u)\left|\varrho^{\prime}\left(a_{1}\right)\right|^{q} d u \\
+\int_{0}^{1} u h(u)\left|\varrho^{\prime}\left(a_{2}\right)\right|^{q} d u+\frac{1}{12} d\left(a_{1}, a_{2}\right) .
\end{gathered}
$$

On the other hand,

$$
\int_{0}^{1} u|1-2 u|^{p} d u=\int_{0}^{1}(1-u)|1-2 u|^{p} d u=\frac{1}{2(p+1)} .
$$

Required inequality (2.6) is obtained by using (2.8), (2.9) and (2.9) in (2.7).

Corollary 2.13. If $\varrho$ is $h$-convex function on $\left[a_{1}, a_{2}\right]$, then

$$
\begin{aligned}
& \left|\frac{\varrho\left(a_{1}\right)+\varrho\left(a_{2}\right)}{2}-\frac{1}{a_{2}-a_{1}} \int_{a_{1}}^{a_{2}} \varrho(x) d x\right| \leq \frac{\left(a_{2}-a_{1}\right)}{2 \sqrt[p]{2(p+1)}} \\
& \times\left\{\left[H_{1}\left|\varrho^{\prime}\left(a_{1}\right)\right|^{q}+H_{2}\left|\varrho^{\prime}\left(a_{2}\right)\right|^{q}\right]^{\frac{1}{q}}+\left[H_{1}\left|\varrho^{\prime}\left(a_{2}\right)\right|^{q}+H_{2}\left|\varrho^{\prime}\left(a_{1}\right)\right|^{q}\right]^{\frac{1}{q}}\right\} .
\end{aligned}
$$

Remark 2.14. If $\varrho$ is $s$-convex function of Breckner type, then we get [15, Theorem 2.2].

Corollary 2.15. If $\varrho$ is s-convex function of Godunova-Levin-Dragomir type on $\left[a_{1}, a_{2}\right]$, then

$$
\begin{gathered}
\left|\frac{\varrho\left(a_{1}\right)+\varrho\left(a_{2}\right)}{2}-\frac{1}{a_{2}-a_{1}} \int_{a_{1}}^{a_{2}} \varrho(x) d x\right| \\
\leq \frac{\left(a_{2}-a_{1}\right)}{2 \sqrt[p]{2(p+1)}}\left[\left(\frac{(1-s)\left|\varrho^{\prime}\left(a_{1}\right)\right|^{q}+\left|\varrho^{\prime}\left(a_{2}\right)\right|^{q}}{(1-s)(2-s)}\right)^{\frac{1}{q}}\right. \\
\left.+\left(\frac{(1-s)\left|\varrho^{\prime}\left(a_{2}\right)\right|^{q}+\left|\varrho^{\prime}\left(a_{1}\right)\right|^{q}}{(1-s)(2-s)}\right)^{\frac{1}{q}}\right]
\end{gathered}
$$


Corollary 2.16. If $\varrho$ is $P$-convex function on $\left[a_{1}, a_{2}\right]$, then

$$
\left|\frac{\varrho\left(a_{1}\right)+\varrho\left(a_{2}\right)}{2}-\frac{1}{a_{2}-a_{1}} \int_{a_{1}}^{a_{2}} \varrho(x) d x\right| \leq \frac{\left(a_{2}-a_{1}\right)}{2 \sqrt[p]{p+1}}\left[\left|\varrho^{\prime}\left(a_{1}\right)\right|^{q}+\left|\varrho^{\prime}\left(a_{2}\right)\right|^{q}\right] .
$$

Remark 2.17. We skip the cases of $\delta$-h-convex functions as they gives similar estimates to $h$-convex functions as discussed earlier.

Corollary 2.18. If $\varrho$ is $(\epsilon, \delta)$-h-convex function on $\left[a_{1}, a_{2}\right]$, then

$$
\begin{gathered}
\left|\frac{\varrho\left(a_{1}\right)+\varrho\left(a_{2}\right)}{2}-\frac{1}{a_{2}-a_{1}} \int_{a_{1}}^{a_{2}} \varrho(x) d x\right| \\
\leq \frac{\left(a_{2}-a_{1}\right)}{2 \sqrt[p]{2(p+1)}}\left\{\left[H_{1}\left|\varrho^{\prime}\left(a_{1}\right)\right|^{q}+H_{2}\left|\varrho^{\prime}\left(a_{2}\right)\right|^{q}+\frac{1}{2}\left[\frac{\epsilon}{6}\left(a_{2}-a_{1}\right)+\delta\right]\right]^{\frac{1}{q}}\right. \\
\left.\quad+\left[H_{1}\left|\varrho^{\prime}\left(a_{2}\right)\right|^{q}+H_{2}\left|\varrho^{\prime}\left(a_{1}\right)\right|^{q}+\frac{1}{2}\left[\frac{\epsilon}{6}\left(a_{2}-a_{1}\right)+\delta\right]\right]^{\frac{1}{q}}\right\} .
\end{gathered}
$$

Corollary 2.19. If $\varrho$ is $(\epsilon, \delta)$-s-convex function of Breckner type on $\left[a_{1}, a_{2}\right]$, then

$$
\begin{gathered}
\left|\frac{\varrho\left(a_{1}\right)+\varrho\left(a_{2}\right)}{2}-\frac{1}{a_{2}-a_{1}} \int_{a_{1}}^{a_{2}} \varrho(x) d x\right| \\
\leq \frac{\left(a_{2}-a_{1}\right)}{2 \sqrt[p]{2(p+1)}}\left[\left(\frac{(1+s)\left|\varrho^{\prime}\left(a_{1}\right)\right|^{q}+\left|\varrho^{\prime}\left(a_{2}\right)\right|^{q}}{(1+s)(2+s)}+\frac{1}{2}\left[\frac{\epsilon}{6}\left(a_{2}-a_{1}\right)+\delta\right]\right)^{\frac{1}{q}}\right. \\
\left.+\left(\frac{(1+s)\left|\varrho^{\prime}\left(a_{2}\right)\right|^{q}+\left|\varrho^{\prime}\left(a_{1}\right)\right|^{q}}{(1+s)(2+s)}+\frac{1}{2}\left[\frac{\epsilon}{6}\left(a_{2}-a_{1}\right)+\delta\right]\right)^{\frac{1}{q}}\right] .
\end{gathered}
$$

Corollary 2.20. If $\varrho$ is $(\epsilon, \delta)$-s-convex function of Godunova-Levin-Dragomir type on $\left[a_{1}, a_{2}\right]$, then

$$
\begin{gathered}
\left|\frac{\varrho\left(a_{1}\right)+\varrho\left(a_{2}\right)}{2}-\frac{1}{a_{2}-a_{1}} \int_{a_{1}}^{a_{2}} \varrho(x) d x\right| \\
\leq \frac{\left(a_{2}-a_{1}\right)}{2 \sqrt[p]{2(p+1)}}\left[\left(\frac{(1-s)\left|\varrho^{\prime}\left(a_{1}\right)\right|^{q}+\left|\varrho^{\prime}\left(a_{2}\right)\right|^{q}}{(1-s)(2-s)}+\frac{1}{2}\left[\frac{\epsilon}{6}\left(a_{2}-a_{1}\right)+\delta\right]\right)^{\frac{1}{q}}\right. \\
\left.+\left(\frac{(1-s)\left|\varrho^{\prime}\left(a_{2}\right)\right|^{q}+\left|\varrho^{\prime}\left(a_{1}\right)\right|^{q}}{(1-s)(2-s)}+\frac{1}{2}\left[\frac{\epsilon}{6}\left(a_{2}-a_{1}\right)+\delta\right]\right)^{\frac{1}{q}}\right] .
\end{gathered}
$$


Corollary 2.21. If $\varrho$ is $(\epsilon, \delta)$-P-convex function on $\left[a_{1}, a_{2}\right]$, then following inequality hold:

$$
\begin{gathered}
\left|\frac{\varrho\left(a_{1}\right)+\varrho\left(a_{2}\right)}{2}-\frac{1}{a_{2}-a_{1}} \int_{a_{1}}^{a_{2}} \varrho(x) d x\right| \leq \frac{\left(a_{2}-a_{1}\right)}{2 \sqrt[2]{p+1}}\left[\left|\varrho^{\prime}\left(a_{1}\right)\right|^{q}+\left|\varrho^{\prime}\left(a_{2}\right)\right|^{q}\right. \\
+\left[\frac{\epsilon}{6}\left(a_{2}-a_{1}\right)+\delta\right]^{\frac{1}{q}} .
\end{gathered}
$$

Corollary 2.22. If $\varrho$ is strongly $h$-convex function on $\left[a_{1}, a_{2}\right]$, then

$$
\begin{gathered}
\left|\frac{\varrho\left(a_{1}\right)+\varrho\left(a_{2}\right)}{2}-\frac{1}{a_{2}-a_{1}} \int_{a_{1}}^{a_{2}} \varrho(x) d x\right| \\
\leq \frac{\left(a_{2}-a_{1}\right)}{2 \sqrt[p]{2(p+1)}}\left[\left(H_{1}\left|\varrho^{\prime}\left(a_{1}\right)\right|^{q}+H_{2}\left|\varrho^{\prime}\left(a_{2}\right)\right|^{q}-\frac{\epsilon}{12}\left(a_{2}-a_{1}\right)^{2}\right)^{\frac{1}{q}}\right. \\
\left.+\left(H_{1}\left|\varrho^{\prime}\left(a_{2}\right)\right|^{q}+H_{2}\left|\varrho^{\prime}\left(a_{1}\right)\right|^{q}-\frac{\epsilon}{12}\left(a_{2}-a_{1}\right)^{2}\right)^{\frac{1}{q}}\right] .
\end{gathered}
$$

Corollary 2.23. If $\varrho$ is strongly s-convex function of Breckner type on $\left[a_{1}, a_{2}\right]$, then

$$
\begin{gathered}
\left|\frac{\varrho\left(a_{1}\right)+\varrho\left(a_{2}\right)}{2}-\frac{1}{a_{2}-a_{1}} \int_{a_{1}}^{a_{2}} \varrho(x) d x\right| \\
\leq \frac{\left(a_{2}-a_{1}\right)}{2 \sqrt[p]{2(p+1)}}\left[\left(\frac{(1+s)\left|\varrho^{\prime}\left(a_{1}\right)\right|^{q}+\left|\varrho^{\prime}\left(a_{2}\right)\right|^{q}}{(1+s)(2+s)}-\frac{\epsilon}{12}\left(a_{2}-a_{1}\right)^{2}\right)^{\frac{1}{q}}\right. \\
\left.+\left(\frac{(1+s)\left|\varrho^{\prime}\left(a_{2}\right)\right|^{q}+\left|\varrho^{\prime}\left(a_{1}\right)\right|^{q}}{(1+s)(2+s)}-\frac{\epsilon}{12}\left(a_{2}-a_{1}\right)^{2}\right)^{\frac{1}{q}}\right] .
\end{gathered}
$$

Corollary 2.24. If $\varrho$ is strongly $s$-convex function of Godunova-LevinDragomir type on $\left[a_{1}, a_{2}\right]$, then

$$
\begin{gathered}
\left|\frac{\varrho\left(a_{1}\right)+\varrho\left(a_{2}\right)}{2}-\frac{1}{a_{2}-a_{1}} \int_{a_{1}}^{a_{2}} \varrho(x) d x\right| \\
\leq \frac{\left(a_{2}-a_{1}\right)}{2 \sqrt[p]{2(p+1)}}\left[\left(\frac{(1-s)\left|\varrho^{\prime}\left(a_{1}\right)\right|^{q}+\left|\varrho^{\prime}\left(a_{2}\right)\right|^{q}}{(1-s)(2-s)}-\frac{\epsilon}{12}\left(a_{2}-a_{1}\right)^{2}\right)^{\frac{1}{q}}\right. \\
\left.+\left(\frac{(1-s)\left|\varrho^{\prime}\left(a_{2}\right)\right|^{q}+\left|\varrho^{\prime}\left(a_{1}\right)\right|^{q}}{(1-s)(2-s)}-\frac{\epsilon}{12}\left(a_{2}-a_{1}\right)^{2}\right)^{\frac{1}{q}}\right] .
\end{gathered}
$$

Corollary 2.25. If $\varrho$ is strongly $P$-convex function on $\left[a_{1}, a_{2}\right]$, then 


$$
\begin{gathered}
\left|\frac{\varrho\left(a_{1}\right)+\varrho\left(a_{2}\right)}{2}-\frac{1}{a_{2}-a_{1}} \int_{a_{1}}^{a_{2}} \varrho(x) d x\right| \leq \frac{\left(a_{2}-a_{1}\right)}{2 \sqrt[p]{p+1}}\left[\left|\varrho^{\prime}\left(a_{1}\right)\right|^{q}+\left|\varrho^{\prime}\left(a_{2}\right)\right|^{q}\right. \\
-\frac{\epsilon}{6}\left(a_{2}-a_{1}\right)^{\frac{1}{q}}
\end{gathered}
$$

Theorem 2.26. Let $\varrho$ be a differentiable function defined on $\left[a_{1}, a_{2}\right]$. If $\left|\varrho^{\prime}\right|^{q}$ for $q \geq 1$, is a modified $(h, d)$-convex function on $\left[a_{1}, a_{2}\right]$, then the following inequality holds:

$$
\begin{gathered}
\left|\frac{\varrho\left(a_{1}\right)+\varrho\left(a_{2}\right)}{2}-\frac{1}{a_{2}-a_{1}} \int_{a_{1}}^{a_{2}} \varrho(x) d x\right| \\
\leq \frac{\left(a_{2}-a_{1}\right)}{2 \sqrt[p]{4}}\left[\left(H_{3}\left|\varrho^{\prime}\left(a_{1}\right)\right|^{q}+H_{4}\left|\varrho^{\prime}\left(a_{2}\right)\right|^{q}+\frac{1}{32} d\left(a_{1}, a_{2}\right)\right)^{\frac{1}{q}}\right. \\
\left.+\left(H_{3}\left|\varrho^{\prime}\left(a_{2}\right)\right|^{q}+H_{4}\left|\varrho^{\prime}\left(a_{1}\right)\right|^{q}+\frac{1}{32} d\left(a_{1}, a_{2}\right)\right)^{\frac{1}{q}}\right]
\end{gathered}
$$

where

$$
\mathrm{H}_{3}=\int_{0}^{1} u|1-2 u| h(u) d u, \quad H_{4}=\int_{0}^{1} u|1-2 u| h(1-u) d u .
$$

Proof. Taking the modulus on both sides of identity (1.11) and applying the improved power-mean integral inequality, we have

$$
\begin{aligned}
& \left|\frac{\varrho\left(a_{1}\right)+\varrho\left(a_{2}\right)}{2}-\frac{1}{a_{2}-a_{1}} \int_{a_{1}}^{a_{2}} \varrho(x) d x\right| \\
& \leq\left(\frac{a_{2}-a_{1}}{2}\right) \int_{0}^{1}|1-2 u|\left|\varrho^{\prime}\left(u a_{1}+(1-u) a_{2}\right)\right| d u \\
& \leq\left(\frac{a_{2}-a_{1}}{2}\right)\left\{\left(\int_{0}^{1}(1-u)|1-2 u| d u\right)^{1-\frac{1}{q}}\right. \\
& \left(\int_{0}^{1}(1-u)|1-2 u|\left|\varrho^{\prime}\left(u a_{1}+(1-u) a_{2}\right)\right|^{q} d u\right)^{\frac{1}{q}}
\end{aligned}
$$




$$
\left.+\left(\int_{0}^{1} u|1-2 u| d u\right)^{1-\frac{1}{q}}\left(\int_{0}^{1} u|1-2 u|\left|\varrho^{\prime}\left(u a_{1}+(1-u) a_{2}\right)\right|^{q} d u\right)^{\frac{1}{q}}\right\} .
$$

Since $\left|\varrho^{\prime}\right|^{q}$ is a modified $(h, d)$-convex function on $\left[a_{1}, a_{2}\right]$, we get

$$
\begin{gathered}
\int_{0}^{1} u|1-2 u|\left|\varrho^{\prime}\left(u a_{1}+(1-u) a_{2}\right)\right|^{q} d u \leq \int_{0}^{1} u|1-2 u| h(u)\left|\varrho^{\prime}\left(a_{1}\right)\right|^{q} d u \\
+\int_{0}^{1} u|1-2 u| h(1-u)\left|\varrho^{\prime}\left(a_{2}\right)\right|^{q} d u+\frac{1}{32} d\left(a_{1}, a_{2}\right)
\end{gathered}
$$

and

$$
\begin{array}{r}
\int_{0}^{1}(1-u)|1-2 u|\left|\varrho^{\prime}\left(u a_{1}+(1-u) a_{2}\right)\right|^{q} d u \leq \int_{0}^{1} u|1-2 u| h(1-u)\left|\varrho^{\prime}\left(a_{1}\right)\right|^{q} d u \\
\quad+\int_{0}^{1} u|1-2 u| h(u)\left|\varrho^{\prime}\left(a_{2}\right)\right|^{q} d u+\frac{1}{32} d\left(a_{1}, a_{2}\right) .
\end{array}
$$

On the other hand,

$$
\int_{0}^{1} u|1-2 u| d u=\int_{0}^{1}(1-u)|1-2 u| d u=\frac{1}{4} .
$$

Required inequality (2.16) is obtained by using (2.18), (2.19) and (2.20) in (2.17).

Corollary 2.27. If $\varrho$ is $h$-convex function on $\left[a_{1}, a_{2}\right]$, then

$$
\left|\frac{\varrho\left(a_{1}\right)+\varrho\left(a_{2}\right)}{2}-\frac{1}{a_{2}-a_{1}} \int_{a_{1}}^{a_{2}} \varrho(x) d x\right|
$$

$\leq \frac{\left(a_{2}-a_{1}\right)}{2 \sqrt[p]{4}}\left[\left(H_{3}\left|\varrho^{\prime}\left(a_{1}\right)\right|^{q}+H_{4}\left|\varrho^{\prime}\left(a_{2}\right)\right|^{q}\right)^{\frac{1}{q}}+\left(H_{3}\left|\varrho^{\prime}\left(a_{2}\right)\right|^{q}+H_{4}\left|\varrho^{\prime}\left(a_{1}\right)\right|^{q}\right)^{\frac{1}{q}}\right]$. 
Remark 2.28. If $\varrho$ is $s$-convex function of Breckner type, then we get [15, Theorem 2.4].

Corollary 2.29. If $\varrho$ is $s$-convex function of Godunova-Levin-Dragomir type on $\left[a_{1}, a_{2}\right]$, then

$$
\begin{gathered}
\left|\frac{\varrho\left(a_{1}\right)+\varrho\left(a_{2}\right)}{2}-\frac{1}{a_{2}-a_{1}} \int_{a_{1}}^{a_{2}} \varrho(x) d x\right| \leq \frac{\left(a_{2}-a_{1}\right)}{2 \sqrt[p]{4}} \\
\times\left\{\left[\frac{(1-s)\left(1-s+2^{s-1}\right)\left|\varrho^{\prime}\left(a_{1}\right)\right|^{q}+\left((5-s) 2^{s-1}-s-1\right)\left|\varrho^{\prime}\left(a_{2}\right)\right|^{q}}{(1-s)(2-s)(3-s)}\right]^{\frac{1}{q}}\right. \\
\left.+\left[\frac{(1-s)\left(1-s+2^{s-1}\right)\left|\varrho^{\prime}\left(a_{2}\right)\right|^{q}+\left((5-s) 2^{s-1}-s-1\right)\left|\varrho^{\prime}\left(a_{1}\right)\right|^{q}}{(1-s)(2-s)(3-s)}\right]^{\frac{1}{q}}\right\} .
\end{gathered}
$$

Corollary 2.30. If $\varrho$ is $P$-convex function on $\left[a_{1}, a_{2}\right]$, then

$$
\left|\frac{\varrho\left(a_{1}\right)+\varrho\left(a_{2}\right)}{2}-\frac{1}{a_{2}-a_{1}} \int_{a_{1}}^{a_{2}} \varrho(x) d x\right| \leq \frac{\left(a_{2}-a_{1}\right)}{4}\left[\left|\varrho^{\prime}\left(a_{1}\right)\right|^{q}+\left|\varrho^{\prime}\left(a_{2}\right)\right|^{q}\right]^{\frac{1}{q}} \text {. }
$$

Remark 2.31. We skip the cases of $\delta$-h-convex functions as they gives similar estimates to $h$-convex functions as discussed earlier.

Corollary 2.32. If $\varrho$ is $(\epsilon, \delta)$-h-convex function on $\left[a_{1}, a_{2}\right]$, then

$$
\begin{gathered}
\left|\frac{\varrho\left(a_{1}\right)+\varrho\left(a_{2}\right)}{2}-\frac{1}{a_{2}-a_{1}} \int_{a_{1}}^{a_{2}} \varrho(x) d x\right| \\
\leq \frac{\left(a_{2}-a_{1}\right)}{2 \sqrt[p]{4}}\left\{\left[H_{3}\left|\varrho^{\prime}\left(a_{1}\right)\right|^{q}+H_{4}\left|\varrho^{\prime}\left(a_{2}\right)\right|^{q}+\frac{1}{4}\left[\frac{\epsilon}{8}\left(a_{2}-a_{1}\right)+\delta\right]\right]^{\frac{1}{q}}\right. \\
\left.+\left[H_{3}\left|\varrho^{\prime}\left(a_{2}\right)\right|^{q}+H_{4}\left|\varrho^{\prime}\left(a_{1}\right)\right|^{q}+\frac{1}{4}\left[\frac{\epsilon}{8}\left(a_{2}-a_{1}\right)+\delta\right]\right]^{\frac{1}{q}}\right\} .
\end{gathered}
$$

Corollary 2.33. If $\varrho$ is $(\epsilon, \delta)$-s-convex function of Breckner type on $\left[a_{1}, a_{2}\right]$, then

$$
\begin{gathered}
\left|\frac{\varrho\left(a_{1}\right)+\varrho\left(a_{2}\right)}{2}-\frac{1}{a_{2}-a_{1}} \int_{a_{1}}^{a_{2}} \varrho(x) d x\right| \leq \frac{\left(a_{2}-a_{1}\right)}{2 \sqrt[p]{4}} \\
\times\left\{\left[\frac{(s+1)\left(s+1+2^{-s-1}\right)\left|\varrho^{\prime}\left(a_{1}\right)\right|^{q}+\left((5+s) 2^{-s-1}+s-1\right)\left|\varrho^{\prime}\left(a_{2}\right)\right|^{q}}{(s+1)(s+2)(s+3)}+\frac{1}{4}\left[\frac{\epsilon}{8}\left(a_{2}-a_{1}\right)+\delta\right]\right]^{\frac{1}{q}}\right. \\
\left.(2.24)+\left[\frac{(s+1)\left(s+1+2^{-s-1}\right)\left|\varrho^{\prime}\left(a_{2}\right)\right|^{q}+\left((5+s) 2^{-s-1}+s-1\right)\left|\varrho^{\prime}\left(a_{1}\right)\right|^{q}}{(s+1)(s+2)(s+3)}+\frac{1}{4}\left[\frac{\epsilon}{8}\left(a_{2}-a_{1}\right)+\delta\right]\right]^{\frac{1}{q}}\right\} .
\end{gathered}
$$


Corollary 2.34. If $\varrho$ is $(\epsilon, \delta)$-s-convex function of Godunova-Levin-Dragomir type on $\left[a_{1}, a_{2}\right]$, then

$$
\begin{gathered}
\left|\frac{\varrho\left(a_{1}\right)+\varrho\left(a_{2}\right)}{2}-\frac{1}{a_{2}-a_{1}} \int_{a_{1}}^{a_{2}} \varrho(x) d x\right| \leq \frac{\left(a_{2}-a_{1}\right)}{2 \sqrt[p]{4}} \\
\times\left\{\left[\frac{(1-s)\left(1-s+2^{s-1}\right)\left|\varrho^{\prime}\left(a_{1}\right)\right|^{q}+\left((5-s) 2^{s-1}-s-1\right)\left|\varrho^{\prime}\left(a_{2}\right)\right|^{q}}{(1-s)(2-s)(3-s)}+\frac{1}{4}\left[\frac{\epsilon}{8}\left(a_{2}-a_{1}\right)+\delta\right]\right]^{\frac{1}{q}}\right. \\
\left.(2.25)+\left[\frac{(1-s)\left(1-s+2^{s-1}\right)\left|\varrho^{\prime}\left(a_{2}\right)\right|^{q}+\left((5-s) 2^{s-1}-s-1\right)\left|\varrho^{\prime}\left(a_{1}\right)\right|^{q}}{(1-s)(2-s)(3-s)}+\frac{1}{4}\left[\frac{\epsilon}{8}\left(a_{2}-a_{1}\right)+\delta\right]\right]^{\frac{1}{q}}\right\} .
\end{gathered}
$$

Corollary 2.35. If $\varrho$ is $(\epsilon, \delta)$-P-convex function on $\left[a_{1}, a_{2}\right]$, then

$$
\begin{gathered}
\left|\frac{\varrho\left(a_{1}\right)+\varrho\left(a_{2}\right)}{2}-\frac{1}{a_{2}-a_{1}} \int_{a_{1}}^{a_{2}} \varrho(x) d x\right| \leq \frac{\left(a_{2}-a_{1}\right)}{4}\left[\left|\varrho^{\prime}\left(a_{1}\right)\right|^{q}+\left|\varrho^{\prime}\left(a_{2}\right)\right|^{q}\right. \\
+\left[\frac{\epsilon}{8}\left(a_{2}-a_{1}\right)+\delta\right]^{\frac{1}{q}} .
\end{gathered}
$$

Corollary 2.36. If $\varrho$ is strongly $h$-convex function on $\left[a_{1}, a_{2}\right]$, then

$$
\begin{gathered}
\left|\frac{\varrho\left(a_{1}\right)+\varrho\left(a_{2}\right)}{2}-\frac{1}{a_{2}-a_{1}} \int_{a_{1}}^{a_{2}} \varrho(x) d x\right| \\
\leq \frac{\left(a_{2}-a_{1}\right)}{2 \sqrt[p]{4}}\left\{\left[H_{3}\left|\varrho^{\prime}\left(a_{1}\right)\right|^{q}+H_{4}\left|\varrho^{\prime}\left(a_{2}\right)\right|^{q}-\frac{\epsilon}{32}\left(a_{2}-a_{1}\right)^{2}\right]^{\frac{1}{q}}\right. \\
\left.+\left[H_{3}\left|\varrho^{\prime}\left(a_{2}\right)\right|^{q}+H_{4}\left|\varrho^{\prime}\left(a_{1}\right)\right|^{q}-\frac{\epsilon}{32}\left(a_{2}-a_{1}\right)^{2}\right]^{\frac{1}{q}}\right\} .
\end{gathered}
$$

Corollary 2.37. If $\varrho$ is strongly s-convex function of Breckner type on $\left[a_{1}, a_{2}\right]$, then

$$
\begin{gathered}
\left|\frac{\varrho\left(a_{1}\right)+\varrho\left(a_{2}\right)}{2}-\frac{1}{a_{2}-a_{1}} \int_{a_{1}}^{a_{2}} \varrho(x) d x\right| \leq \frac{\left(a_{2}-a_{1}\right)}{2 \sqrt[p]{4}} \\
\times\left\{\left[\frac{(s+1)\left(s+1+2^{-s-1}\right)\left|\varrho^{\prime}\left(a_{1}\right)\right|^{q}+\left((5+s) 2^{-s-1}+s-1\right)\left|\varrho^{\prime}\left(a_{2}\right)\right|^{q}}{(s+1)(s+2)(s+3)}-\frac{\epsilon}{32}\left(a_{2}-a_{1}\right)^{2}\right]^{\frac{1}{q}}\right. \\
\left.(2.27) \quad+\left[\frac{(s+1)\left(1+s+2^{-s-1}\right)\left|\varrho^{\prime}\left(a_{2}\right)\right|^{q}+\left((5+s) 2^{-s-1}+s-1\right)\left|\varrho^{\prime}\left(a_{1}\right)\right|^{q}}{(s+1)(s+2)(s+3)}-\frac{\epsilon}{32}\left(a_{2}-a_{1}\right)^{2}\right]^{\frac{1}{q}}\right\} .
\end{gathered}
$$


Corollary 2.38. If $\varrho$ is strongly $s$-convex function of Godunova-LevinDragomir type on $\left[a_{1}, a_{2}\right]$, then

$$
\begin{gathered}
\left|\frac{\varrho\left(a_{1}\right)+\varrho\left(a_{2}\right)}{2}-\frac{1}{a_{2}-a_{1}} \int_{a_{1}}^{a_{2}} \varrho(x) d x\right| \leq \frac{\left(a_{2}-a_{1}\right)}{2 \sqrt[p]{4}} \\
\times\left\{\left[\frac{(1-s)\left(1-s+2^{s-1}\right)\left|\varrho^{\prime}\left(a_{1}\right)\right|^{q}+\left((5-s) 2^{s-1}-s-1\right)\left|\varrho^{\prime}\left(a_{2}\right)\right|^{q}}{(1-s)(2-s)(3-s)}-\frac{\epsilon}{32}\left(a_{2}-a_{1}\right)^{2}\right]^{\frac{1}{q}}\right. \\
\left.\quad+\left[\frac{(1-s)\left(1-s+2^{s-1}\right)\left|\varrho^{\prime}\left(a_{2}\right)\right|^{q}+\left((5-s) 2^{s-1}-s-1\right)\left|\varrho^{\prime}\left(a_{1}\right)\right|^{q}}{(1-s)(2-s)(3-s)}-\frac{\epsilon}{32}\left(a_{2}-a_{1}\right)^{2}\right]^{\frac{1}{q}}\right\} .
\end{gathered}
$$

Corollary 2.39. If $\varrho$ is strongly $P$-convex function on $\left[a_{1}, a_{2}\right]$, then

$$
\begin{gathered}
\mid \frac{\varrho\left(a_{1}\right)+\varrho\left(a_{2}\right)}{2}-\frac{1}{a_{2}-a_{1}} \int_{a_{1}}^{a_{2}} \varrho(x) d x \\
\leq \frac{\left(a_{2}-a_{1}\right)}{4}\left[\left|\varrho^{\prime}\left(a_{1}\right)\right|^{q}+\left|\varrho^{\prime}\left(a_{2}\right)\right|^{q}-\frac{\epsilon}{8}\left(a_{2}-a_{1}\right)^{2}\right]^{\frac{1}{q}} .
\end{gathered}
$$

\section{Applications to numerical integration}

In this section, we give some new error estimates for the trapezoidal quadrature formula by using inequalities developed in the Section 2. Assume that $P$ is a partition of the interval $\left[a_{1}, a_{2}\right]$, i.e.,

$$
P: a_{1}=x_{0}<x_{1}<x_{2}<\ldots<x_{n-1}<x_{n}=a_{2} .
$$

Trapezoidal quadrature formula is defined by

$$
\int_{a_{1}}^{a_{2}} \varrho(x) d x=T(\varrho, P)+E(\varrho, P)
$$

where

$$
T(\varrho, P)=\sum_{i=0}^{n-1}\left[\frac{\varrho\left(x_{i}\right)+\varrho\left(x_{i+1}\right)}{2}\right] h_{i}
$$

is the trapezoidal version for $h_{i}=\left(x_{i+1}-x_{i}\right)$ and $i=0,1, \ldots, n-1$, and $E(\varrho, P)$ is denote their associated approximation error. 
Proposition 3.1. Let $\varrho:\left[a_{1}, a_{2}\right] \longrightarrow \mathbf{R}$ be a differentiable function on $\left(a_{1}, a_{2}\right)$, where $a_{1}<a_{2}$. If $\left|\varrho^{\prime}\right|^{q}$ is strongly s-convex of Godunova-LevinDragomir type on $\left[a_{1}, a_{2}\right]$ for $q>1$ and $\frac{1}{p}+\frac{1}{q}=1$, then the following inequality holds:

$$
\begin{gathered}
|E(\varrho, P)| \leq \frac{1}{2 \sqrt[p]{2(p+1)}} \sum_{i=0}^{n-1} h_{i}^{2} \\
\times\left[\left(\frac{(1-s)\left|\varrho^{\prime}\left(x_{i}\right)\right|^{q}+\left|\varrho^{\prime}\left(x_{i+1}\right)\right|^{q}}{(1-s)(2-s)}-\frac{\epsilon}{12} h_{i}^{2}\right)^{\frac{1}{q}}+\left(\frac{(1-s)\left|\varrho^{\prime}\left(x_{i+1}\right)\right|^{q}+\left|\varrho^{\prime}\left(x_{i}\right)\right|^{q}}{(1-s)(2-s)}-\frac{\epsilon}{12} h_{i}^{2}\right)^{\frac{1}{q}}\right] .
\end{gathered}
$$

Proof. Applying inequality (3.1) in Corollary 2.24 on the subintervals $\left[x_{i}, x_{i+1}\right](i=0, \ldots, n-1)$ of the partition $P$, we have

$$
\begin{gathered}
\left|\frac{\varrho\left(x_{i}\right)+\varrho\left(x_{i+1}\right)}{2}-\frac{1}{x_{i+1}-x_{i}} \int_{x_{i}}^{x_{i+1}} \varrho(x) d x\right| \leq \frac{\left(x_{i+1}-x_{i}\right)}{2 \sqrt[p]{2(p+1)}} \\
\quad \times\left[\left(\frac{(1-s)\left|\varrho^{\prime}\left(x_{i}\right)\right|^{q}+\left|\varrho^{\prime}\left(x_{i+1}\right)\right|^{q}}{(1-s)(2-s)}-\frac{\epsilon}{12} h_{i}^{2}\right)^{\frac{1}{q}}\right. \\
\left.+\left(\frac{(1-s)\left|\varrho^{\prime}\left(x_{i+1}\right)\right|^{q}+\left|\varrho^{\prime}\left(x_{i}\right)\right|^{q}}{(1-s)(2-s)}-\frac{\epsilon}{12} h_{i}^{2}\right)^{\frac{1}{q}}\right] .
\end{gathered}
$$

Hence from (3.3), we get

$$
\begin{aligned}
|E(\varrho, P)| & =\left|\int_{a_{1}}^{a_{2}} \varrho(x) d x-T(\varrho, P)\right| \\
& \leq\left|\sum_{i=0}^{n-1}\left\{\int_{x_{i}}^{x_{i+1}} \varrho(x) d x-\frac{\varrho\left(x_{i}\right)+\varrho\left(x_{i+1}\right)}{2} h_{i}\right\}\right| \\
& \leq \sum_{i=0}^{n-1}\left|\left\{\int_{x_{i}}^{x_{i+1}} \varrho(x) d x-\frac{\varrho\left(x_{i}\right)+\varrho\left(x_{i+1}\right)}{2} h_{i}\right\}\right| \\
\leq & \frac{1}{2 \sqrt[p]{2(p+1)}} \sum_{i=0}^{n-1} h_{i}^{2} \\
& \times\left[\left(\frac{(1-s)\left|\varrho^{\prime}\left(x_{i}\right)\right|^{q}+\left|\varrho^{\prime}\left(x_{i+1}\right)\right|^{q}}{(1-s)(2-s)}-\frac{\epsilon}{12} h_{i}^{2}\right)^{\frac{1}{q}}\right.
\end{aligned}
$$




$$
\left.+\left(\frac{(1-s)\left|\varrho^{\prime}\left(x_{i+1}\right)\right|^{q}+\left|\varrho^{\prime}\left(x_{i}\right)\right|^{q}}{(1-s)(2-s)}-\frac{\epsilon}{12} h_{i}^{2}\right)^{\frac{1}{q}}\right] .
$$

The proof of Proposition 3.1 is completed.

Proposition 3.2. Let $\varrho:\left[a_{1}, a_{2}\right] \longrightarrow \mathbf{R}$ be a differentiable function on $\left(a_{1}, a_{2}\right)$, where $a_{1}<a_{2}$. If $\left|\varrho^{\prime}\right|^{q}$ is strongly s-convex of Godunova-LevinDragomir type on $\left[a_{1}, a_{2}\right]$ for $q \geq 1$, then the following inequality holds:

$$
\begin{gathered}
|E(f, Q)| \leq \frac{1}{2 \sqrt[p]{4}} \sum_{i=0}^{n-1} h_{i}^{2} \\
\times\left\{\left[\frac{(1-s)\left(1-s+2^{s-1}\right)\left|\varrho^{\prime}\left(x_{i}\right)\right|^{q}+\left((5-s) 2^{s-1}-s-1\right)\left|\varrho^{\prime}\left(x_{i+1}\right)\right|^{q}}{(1-s)(2-s)(3-s)}-\frac{\epsilon}{32} h_{i}^{2}\right]^{\frac{1}{q}}\right. \\
\left.+\left[\frac{(1-s)\left(1-s+2^{s-1}\right)\left|\varrho^{\prime}\left(x_{i+1}\right)\right|^{q}+\left((5-s) 2^{s-1}-s-1\right)\left|\varrho^{\prime}\left(x_{i}\right)\right|^{q}}{(1-s)(2-s)(3-s)}-\frac{\epsilon}{32} h_{i}^{2}\right]^{\frac{1}{q}}\right\} .
\end{gathered}
$$

Proof. The proof is analogous as to that of Proposition 3.1 but use inequality (2.28) in Corollary 2.38.

Remark 3.3. By using Theorem 2.1, for appropriate choices of function $\varrho$ and function $h(u)=u, u^{-1}, u^{s}, u^{-s}, u(1-u), \frac{\sqrt{u}}{2 \sqrt{1-u}}$ and $h(u)=1$, we can obtain some new integral inequalities using special means, such as arithmetic, geometric, logarithmic etc. Also, applying our Theorems 2.12 and 2.26 for above suitable functions $h$, we can deduce some new integral inequalities using ideas and techniques of Propositions 3.1 and 3.2. We omit their proofs and the details are left to the interested reader.

\section{Conclusion}

In this study, by applying Hölder-Íşcan integral inequality three inequalities of Hermite-Hadamard and Hadamard integral type for modified $(h, d)$ convex functions have been provided. Various special cases including classes for instance, $h$-convex, $s$-convex function of Breckner and Godunova-LevinDragomir and strong versions of the aforementioned types of convex functions have been obtained. Also, some new applications to special means and error estimations of presented results can be analyzed by interested readers but we omit here their proofs. We hope that current work will attract the attention of researchers working in mathematical analysis, fractional calculus, $(p, q)$-calculus and other related fields. 


\section{References}

[1] H. Angulo, J. Giménez, A. M. Moros, and K. Nikodem, "On strongly h-convex functions", Annals of functional analysis, vol. 2, no. 2, pp. 85-91, 2011, doi: 10.15352/ afa/ 1399900197

[2] P. Agarwal, "Some inequalities involving Hadamard-type k-fractional integral operators", Mathematical methods in the applied sciences, vol. 40, no. 11, pp. 3882-3891, 2017, doi: 10.1002/ mma.4270

[3] P. Agarwal, M. Jleli, and M. Tomar, "Certain Hermite-Hadamard type inequalities via generalized k-fractional integrals", Journal of inequalities and applications, vol. 2017, no. 1, 2017, doi: 10.1186/ s13660-017-1318-y

[4] S. Mohammadi Aslani, M. Rostamian Delavar, and S M. Vaezpour, "Inequalities of Féjer type related to generalized convex functions", International journal of analysis and applications, vol. 6, no. 1, pp. 38-49, 2018, doi: 10.28924/ 2291-8639-16-2018-38

[5] P. Burai, and A. Házy, "On approximately h-convex function”, Journal of convex analysis, vol. 18, no. 2, pp. 447-454, 2011. [On line]. Available: https:/ / bit.ly/ 2OnVeiz

[6] F. X. Chen and S. H. Wu, "Several complementary inequalities to inequalities of Hermite-Hadamard type for s-convex functions", Journal of nonlinear science and applications, vol. 9, no. 2, pp. 705-716, 2016. [On line]. Available: https:/ / bit.ly/ 30cWQyg

[7] Y. M. Chu, M. A. Khan, T. U. Khan, and T. Ali, "Generalizations of Hermite-Hadamard type inequalities for MT-convex functions", Journal of nonlinear science and applications, vol. 9, no. 6, pp. 4305-4316, 2016. [On line]. Available: https:/ / bit.ly/ 3c23lcQ

[8] M. R. Delavar and M. De La Sen, "Some generalizations of HermiteHadamard type inequalities", SpringerPlus, vol. 5, no. 1, Art ID. 1661, 2016, doi: 10.1186/ s40064-016-3301-3

[9] S. S. Dragomir and R. P. Agarwal, "Two inequalities for differentiable mappings and applications to special means of real numbers and to trapezoidal formula", Applied mathematics letters, vol. 11, no. 5, pp. 91-95, 1998, doi: 10.1016/ S0893-9659(98)00086-X

[10] S. S. Dragomir and C. E. M. Pearce, Selected topics on HermiteHadamard inequalities and applications. Melbourne, VIC: Victoria University, 2000. [On line]. Available: https:/ / bit.ly/ 2OmQMR7

[11] A. Ekinci and M. E. Özdemir, "Some new integral inequalities via Riemann-Liouville integral operators", Applied and computational mathematics, vol. 18, no. 3, pp. 288-295, 2019. 
[12] G. Farid and A. U. Rehman, "Generalizations of some integral inequalities for fractional integrals", Annales mathematicae silesianae, vol. 32, no. 1, pp. 201-214, 2018, doi: 10.1515/ amsil-2017-0010

[13] D. H. Hyers and S. M. Ulam, "Approximately convex functions", Proceedings of the American Mathematical Society, vol. 3, no. 5, pp. 821-821, 1952, doi: 10.1090/ S0002-9939-1952-0049962-5

[14] Í. Íşcan, "New refinements for integral and sum forms of Hölder inequality", Journal of inequalities and applications, vol. 2019, no. 1, pp. Art ID. 304, 2019, doi: 10.1186/ s13660-019-2258-5

[15] Ś. Özcan and Í. Íşcan, "Some new Hermite-Hadamard type inequalities for s-convex functions and their applications", Journal of inequalities and applications, vol. 2019, Art. ID. 201, 2019, doi: 10.1186/ s13660-019-2151-2

[16] M. Kadakal, M., Í. Íşcan, H. Kadakal, and K. Bekar, "On improvements of some integral inequalities", ResearchGate, Jan. 2019, doi: 10.13140/ RG.2.2.15052.46724

[17] A. Kashuri and R. Liko, "Some new Hermite-Hadamard type inequalities and their applications", Studia scientiarum mathematicarum hungarica, vol. 56, no. 1, pp. 103-142, 2019, doi: 10.1556/ 012.2019.56.1.1418

[18] M. A. Khan, Y.-M. Chu, A. Kashuri, R. Liko, and G. Ali, "Conformable fractional integrals versions of Hermite-Hadamard inequalities and their generalizations", Journal of function spaces, vol. 2018, Art ID. 6928130, 2018, doi: 10.1155/2018/6928130

[19] W. Liu, W. Wen, and J. Park, "Hermite-Hadamard type inequalities for MT-convex functions via classical integrals and fractional integrals", Journal of nonlinear sciences and applications, vol. 09, no. 03, pp. 766-777, 2016, doi: 10.22436/jnsa.009.03.05

[20] M. V. Mihai, "Some Hermite-Hadamard type inequalities via RiemannLiouville fractional calculus", Tamkang journal of mathematics, vol. 44, no. 4, pp. 411-416, 2013, doi: 10.5556/j.tkjm.44.2013.1218

[21] S. Mubeen and G. M. Habibullah, "k-Fractional integrals and applications", International journal of contemporary mathematical sciences, vol. 7, no. 1-4, pp. 89-94, 2012. [On line]. Available: https:/ / bit.ly/ 3bfZqdv

[22] O. Omotoyinbo and A. Mogbodemu, "Some new Hermite-Hadamard integral inequalities for convex functions", International journals of science and innovation technology, vol. 1, no. 1, pp. 1-12, 2014.

[23] M. E. Özdemir, S. S. Ragomir, and Ç. Yildiz, "The Hadamard inequality for convex function via fractional integrals", Acta mathematica scientia, vol. 33, no. 5, pp. 1293-1299, 2013, doi: 10.1016/ S0252-9602(13) 60081-8 
[24] Z. Páles, "On approximately convex functions", Proceedings of the American Mathematical Society, vol. 131, no. 1, pp. 243-252, 2002, doi: 10.1090/ S0002-9939-02-06552-8

[25] M. Z. Sarikaya, A, Saglam, and H. Yildirim, "On some Hadamard type inequalities for h-convex functions", Journal of mathematical inequalities, vol. 2, no. 3, pp. 335-341, 2008, doi: 10.7153/jmi-02-30

[26] M. Z. Sarikaya, and H. Yildirim, "On generalization of the Riesz potential", Indian journal of mathematics and mathematical sciences, vol. 3, no. 2, pp. 231-235, 2007. [On line]. Available: https:/ / bit.ly/ 3eeIPs6

[27] E. Set, M. A. Noor, M. U. Awan, and A. Gözpinar, "Generalized HermiteHadamard type inequalities involving fractional integral operators", Journal of inequalities and applications, vol. 2017, no. 1, Art ID. 169, 2017, doi: 10.1186/ s13660-017-1444-6

[28] E. Set, A. O. Akdemir, and B. Çelik, "On generalization of Fejér type inequalities via fractional integral operator", Filomat, vol. 32, no. 16, pp. 5537-5547, 2018, doi: 10.2298/ FIL1816537S

[29] E. Set, A. O. Akdemir, and E. A. Alan, "Hermite-Hadamard and HermiteHadamard-Fejér type inequalities involving fractional integral operators", Filomat, vol. 33, no. 8, pp. 2367-2380, 2019, doi: 10.2298/ FIL1908367S

[30] H. Wang, T. Du, and Y. Zhang, "k-fractional integral trapezium-like inequalities through ( $h, \mathrm{~m})$-convex and $(\alpha, \mathrm{m})$-convex mappings", Journal of inequalities and applications, vol. 2017, no. 1, Art ID. 311, 2017, doi: 10.1186/ s13660-017-1586-6

[31] B. Y. Xi and F. Qi, "Some integral inequalities of Hermite-Hadamard type for convex functions with applications to means", Journal of function spaces, 2012, Art. ID. 980438, 2012, doi: 10.1155/ 2012/ 980438. 